\title{
The Phase Diagram of Random Heteropolymers
}

\author{
A. Montanari ${ }^{1}$, M. Müller ${ }^{2}$, M. Mézard ${ }^{2}$ \\ ${ }^{1}$ Laboratoire de Physique Théorique de l'Ecole Normale Supérieure* and \\ ${ }^{2}$ Laboratoire de Physique Théorique et Modèles Statistiques, \\ Université Paris-Sud, bâtiment 100, F-91405 Orsay, France.
}

(Dated: November 10, 2018)

\begin{abstract}
We propose a new analytic approach to study the phase diagram of random heteropolymers, based on the cavity method. For copolymers we analyze the nature and phenomenology of the glass transition as a function of sequence correlations. Depending on these correlations, we find that two different scenarios for the glass transition can occur. We show that, beside the much studied possibility of an abrupt freezing transition at low temperature, the system can exhibit, upon cooling, a first transition to a soft glass phase with fully broken replica symmetry and a continuously growing degree of freezing as the temperature is lowered.

PACS numbers: 81.05.Lg, 64.70.Pf, 36.20.Ey
\end{abstract}

The statistical mechanics of random heteropolymers has attracted a lot of interest in the past two decades, mainly for its close relation to the long standing problem of protein folding [1], but also because of its applications to material sciences [2]. It has soon been recognized that the energy landscape and low temperature physics of heteropolymers bear a strong resemblance with that of spin glasses, and many theoretical concepts have been fruitfully adapted to that particular class of disordered systems [3, 4].

A popular guiding idea in this field was the conjecture that typical low energy configurations of a random heteropolymer behave as independent energy levels 5 as it happens in the random energy model (REM) [6]. Mean field replica calculations corroborate such a discontinuous glass transition with one step of replica symmetry breaking, both for random bond interactions [7] and for copolymers [8]. However, the applicability of the simple REM-like picture to heteropolymers and proteins has been questioned [9]. The numerical study of selfconsistent dynamical equations for short chains [10] hints at a more refined scenario which we shall compare with our own results below.

The polymer freezing manifests itself in persisting correlations between two times $t_{w}$ and $t_{w}+t$, after the molecule has been prepared in a random conformation at time 0 [1]. The following family of correlation functions for a chain of length $N$ proves very useful [12]: $C_{l}\left(t, t_{w}\right)=N^{-1} \sum_{i}\left\langle\Delta \boldsymbol{r}_{i, l}(t) \cdot \Delta \boldsymbol{r}_{i, l}\left(t_{w}\right)\right\rangle$. Here $\Delta \boldsymbol{r}_{i, l}=\boldsymbol{r}_{i+l}-\boldsymbol{r}_{i}$ and $\boldsymbol{r}_{i}$ is the spatial position of the $i$-th monomer of the chain. Within the glassy phase, these correlation functions should exhibit a plateau at $q_{l}=$ $\lim _{t \rightarrow \infty} \lim _{t_{w} \rightarrow \infty} C_{l}\left(t, t_{w}\right)$ ( $t$ and $t_{w}$ are much smaller than the equilibration time $\left.\tau_{\text {eq }}\right)$.

The REM-like scenario predicts an abrupt freezing in a

*UMR 8549, Unité Mixte de Recherche du Centre National de la Recherche Scientifique et de l' Ecole Normale Supérieure. metastable conformation, implying a discontinuous jump of the plateau height $q_{l}$ from zero to a finite value at the glass transition. However, many other disordered systems, like spin glasses, exhibit a continuous glass transition (in the "overlap" parameter, $q_{l}$ ). This has recently been predicted for the random amphiphilic chain [12], as does our analysis for a certain class of correlated sequences. The folding properties of a given heteropolymer will be crucially affected by the type of glass transition it undergoes, since the dynamics (e.g., the aging behavior) are rather different in these two scenarios 11].

Apart from numerical simulations, most of our fundamental knowledge about heteropolymers relies on two main analytical methods: the replica approach, with some kind of Gaussian variational Ansatz [7], and the use of dynamical equations, with some self-consistent closure approximation [10, 13]. Considering that these methods use some rather severe approximations which are not really independent from each other, it is clearly useful to have a new analytic method emerging. We propose here such a new approach, based on the cavity method 14], which is an extension of the classical Bethe approximation to frustrated systems. It is the simplest mean-field approximation that handles exactly the short distance correlations [15], and in this respect it is very different from the usual replica approach. Moreover, as we will see below, the existence of a non-trivial statistical mechanics model on a 'Bethe lattice' for which the method is exact is very helpful. We expect the cavity approximation to be faithful as long as crystalline, or more complex, spatially ordered structures are suppressed by the randomness of the chain sequence.

We show that for fixed monomer-monomer interactions, both types of glass transition can be found. Depending on the correlations in the chain sequence, one finds either a transition to a 'frozen glass' phase, with a jump in $q_{l}$ (we shall call $T_{d}=1 / \beta_{d}$ the corresponding transition temperature), or a continuous transition to a 'soft glass' phase, where $q_{l}$ develops continuously 
from zero (we denote by $\beta_{i}$ the inverse critical temperature in this case), see Figs. 1 and 2 The predicted soft glass phase bears some resemblance with the intermediate glass phase found in the dynamic approaches [10].

Consider a lattice polymer whose conformation is given by a self-avoiding walk. Two monomers interact with an energy $e_{\sigma, \sigma^{\prime}}$ if they occupy two neighboring sites $i$ and $j$. Here $\sigma, \sigma^{\prime} \in\{1,-1\} \leftrightarrow\{A, B\}$ denote the monomer types. We will restrict to the symmetric case $e_{A A}=e_{B B}=-e_{A B}$ and only consider neutral polymers (equal number of $A$ 's and $B$ 's). The case $e_{A A}=-1$ is close to the popular HP-model for proteins where hydrophobic and polar constituents tend to cluster, while $e_{A A}=1$ corresponds to charged polymers (ampholytes) with short-range interactions.

On a lattice with $V$ sites, we consider the polymer in the grand canonical ensemble with a chemical potential $\mu$ per monomer. One can identify two phases depending on the scaling of the average length $\langle N\rangle$ of the polymer [15]. For small $\mu\left(\mu<\mu_{c}(T)\right)$ there exists an infinitely diluted phase where $\langle N\rangle / V \rightarrow 0$ as $V \rightarrow \infty$. For $\mu>\mu_{c}$, there is a dense phase with a finite $\langle N\rangle / V$. To describe a polymer in equilibrium with the solvent the chemical potential has to be adjusted to the critical value $\mu_{c}$. The $\Theta$-point where the polymer collapses to the liquid globule occurs as a tricritical point on this line [16], see Fig. [1]

Before explaining the cavity approach, we notice that its approximations become exact on a 'Bethe lattice' whose appropriate definition for a frustrated system is a random graph with $N$ vertices and fixed connectivity (taken equal to $k+1 \equiv 6$ here to mimic cubic lattices) 14]. Since the size of typical loops in such a graph diverges as $O(\log (N))$, the graph is locally tree-like, allowing for iterative solutions of the statistical physics problem.

For technical reasons we work with a periodic sequence of monomers with period $L$ and take the limit $L \rightarrow \infty$ in the end. Let us now consider an oriented edge of the graph, going from a 'root' site $\mathrm{R}$ to another site $\mathrm{S}$. It can be in one of the following states: $(0)$ : neither root nor edge are occupied; $(+, a)$ or $(-, a)$ : the root is occupied by monomer $a \in[1, L]$ and the backbone continues on the edge, the sign \pm indicating that $\mathrm{S}$ is occupied by monomer $a \pm 1 ;(2 a)$ : the root is occupied by monomer $a$, but the edge is free. We now exploit the local tree-like structure to recursively express the (Gibbs) probability $p_{\alpha}^{(0)}$ of finding the oriented edge ' 0 ' in the conformation $\alpha \in\{0,(+, a),(-, a), 2 a\}$ in terms of the corresponding probabilities $p_{\alpha^{\prime}}^{(i)}$ on the remaining edges $(i=1, \ldots, k)$ that are connected to the root of the edge ' 0 ':

$$
p_{0}^{(0)}=C^{-1} \prod_{i=1}^{k}\left(p_{0}^{(i)}+\sum_{a^{\prime}=1}^{L} p_{2 a^{\prime}}^{(i)}\right)
$$
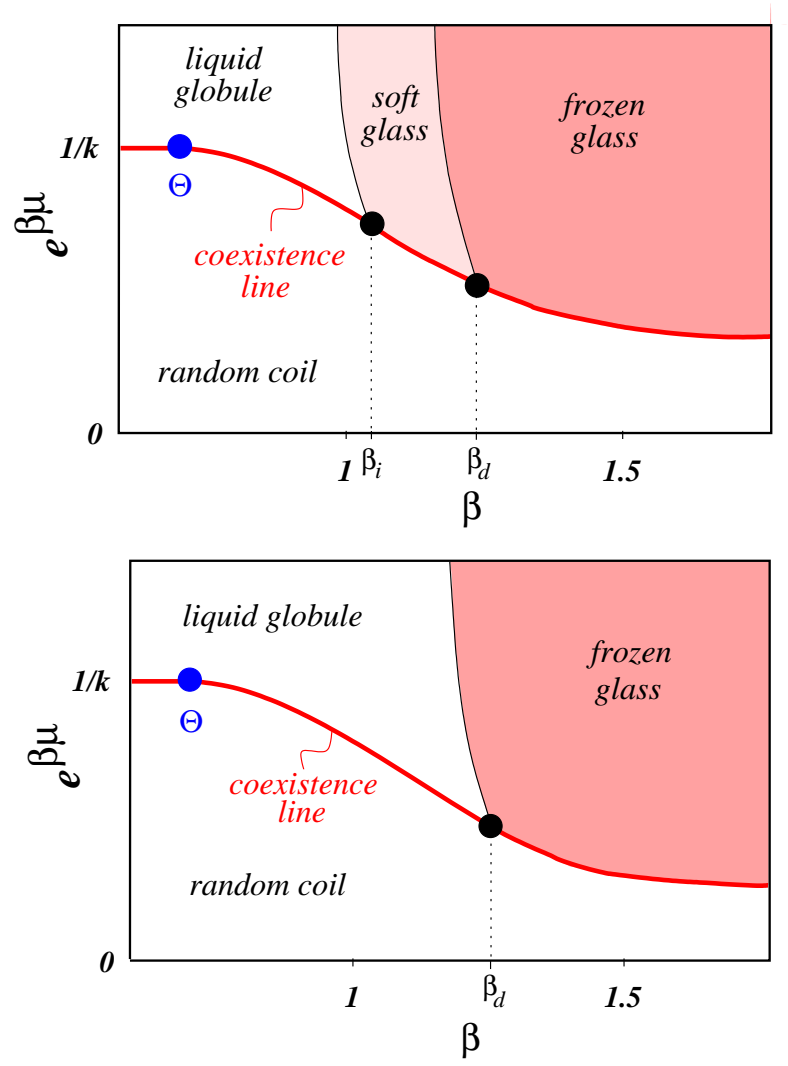

FIG. 1: The phase diagram in the grand canonical ensemble. An unconstrained polymer lives on the coexistence line $\left(\mu=\mu_{c}(T)\right)$. In correlated HP-polymers and anticorrelated ampholytes (top) a continuous glass transition occurs at the liquid instability. It is followed by a discontinuous freezing transition. Oppositely correlated sequences (bottom) exhibit a direct freezing transition from the liquid globule.

$$
\begin{aligned}
p_{ \pm, a}^{(0)} & =C^{-1} e^{\beta \mu} \sum_{i=1}^{k} p_{ \pm, a \mp 1}^{(i)} \prod_{j \neq i}^{k} \psi_{a}^{(j)} \\
p_{2 a}^{(0)} & =C^{-1} e^{\beta \mu} \sum_{i_{1} \neq i_{2}}^{k} p_{+, a-1}^{\left(i_{1}\right)} p_{-, a+1}^{\left(i_{2}\right)} \prod_{j \neq i_{1}, i_{2}}^{k} \psi_{a}^{(j)},
\end{aligned}
$$

where $C$ is a normalization and we have introduced $\psi_{a}^{(j)}=p_{0}^{(i)}+\sum_{a^{\prime}=1}^{L} p_{2 a^{\prime}}^{(j)} e^{-\beta e_{\sigma_{a}, \sigma} a^{\prime}}$.

The recursion is exact on a tree, but it also holds asymptotically on a large $N$ Bethe lattice, in the liquid phase or inside a pure state where the probabilities on the $k$ parent links are uncorrelated.

The liquid phase is described by a fixed point $p_{\alpha}^{(i)} \equiv p_{\alpha}^{*}$ of Eqs. (13). The liquid free energy $\phi_{\text {liq }}$ is obtained as a sum of site and edge contributions which are expressed in terms of $\left\{p_{\alpha}^{*}\right\}$ following the general prescription of Ref. 14]. It turns out that the liquid globule of neutral copolymers bears no trace of disorder since $\phi_{\text {liq }}$ is independent of the sequence.

For any heterogeneous sequence the entropy of the liquid solution turns negative at sufficiently low temper- 
atures, indicating the existence of a glass phase which breaks the translational invariance. The latter is also clearly observed in Monte Carlo simulations both on the Bethe and the cubic 3d lattice 17. To treat the glass phase, the above framework has to be extended. We assume that below the glass transition the phase space splits into a large number of pure states. Restricting the Gibbs measure to a state $\gamma$ gives rise to probabilities $p_{\alpha}^{(i ; \gamma)}$, and we again obtain a closed recursion relation if we look at the distribution of local probabilities over all pure states [14], $\rho(\mathbf{p})=\mathcal{N}^{-1} \sum_{\gamma} w_{\gamma} \delta\left(\mathbf{p}-\mathbf{p}^{(i ; \gamma)}\right)$. It will not depend on the site $i$ since the graph is regular. The cavity recursion then reads

$$
\begin{gathered}
\rho(\mathbf{p})=\frac{1}{\mathcal{Z}} \int \prod_{i=1}^{k} \rho\left(\mathbf{p}^{(i)}\right) d \mathbf{p}^{(i)} \delta\left(\mathbf{p}-\mathbf{p}^{(0)}\left[\mathbf{p}^{(1)}, \ldots, \mathbf{p}^{(k)}\right]\right) \\
e^{-m \beta \Delta f\left[\mathbf{p}^{(1)}, \ldots, \mathbf{p}^{(k)}\right]}
\end{gathered}
$$

where $\mathbf{p}^{(0)}\left[\mathbf{p}^{(1)}, \ldots, \mathbf{p}^{(k)}\right]$ is the pure state recursion (13).

We have introduced in (4) a reweighting with respect to the free energy change $\Delta f$ corresponding to the addition of the site 0 , in order to take into account the exponential increase of the number of pure states as a function of the total free energy. $\Delta f$ is related to the normalization factor in (13) by $\exp (-\beta \Delta f)=C$. The reweighting depends on $m \in[0,1]$ which is the cavity ana$\log$ of the breakpoint parameter appearing in Parisi's onestep replica symmetry breaking scheme. As explained in [14, 17], one can write down an $m$-dependent free energy $\phi_{1}(m)$, and the dominant metastable states correspond to $m^{*}$ which maximizes $\phi_{1}(m)$.

The local stability of the liquid phase with respect to the glass transition can be studied by computing appropriately defined glass susceptibilities. The simplest one can be written by associating to each site $i$ of the lattice a 'spin' $s_{i}$. We define $s_{i}=0$ if $i$ is empty and $s_{i}=\sigma$, if $i$ is occupied by a monomer of type $\sigma$. The susceptibility is given by $\chi_{g}=V^{-1} \sum_{i j} \overline{\left\langle s_{i} s_{j}\right\rangle^{2}}$. A divergence of $\chi_{g}$ signals the appearance of a glass phase.

The instability of the liquid can be analyzed for arbitrary sequences of monomers. It occurs at the temperature $\beta_{i}$ determined by

$$
\frac{1+z_{2}\left(\beta_{i}\right) \cosh \left(\beta_{i}\right)}{z_{2}\left(\beta_{i}\right) \sinh \left(\beta_{i}\right)}=-k^{1 / 2}+\frac{2(k-1) Q_{\mathrm{seq}}}{k^{1 / 2}\left(1-k^{-L / 2}\right)},
$$

where $z_{2}\left(\beta_{i}\right)=L p_{2}^{*} / p_{0}^{*}$ is independent of the sequence [17]. The latter only enters through the term $Q_{\text {seq }}=\sum_{b=0}^{L-1} q_{b}( \pm \sqrt{k})^{-b}$ where $q_{b}=1 / L \sum_{a=1}^{L} \sigma_{a} \sigma_{a+b}$ is the sequence autocorrelation function and the upper/lower sign corresponds to the HP-model and the ampholyte, respectively. Equation (5) can be regarded as the generalization of a classic result by de Gennes [18] to strongly frustrated systems.

In Fig. 2 we plot the local instability $\beta_{i}$ obtained from Eq. (5]) as a function of sequence correlations in the simple case of Markovian chains described by the probability

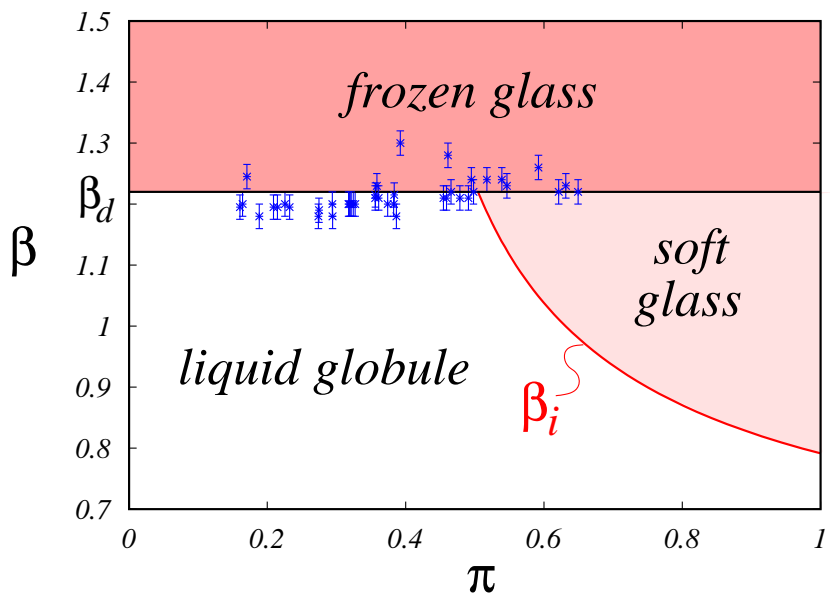

FIG. 2: The phase diagram as a function of sequence correlations $\pi$ for HP polymers. At high temperature (small $\beta$ ) the polymer is found in a liquid globule phase. For correlated sequences $(\pi \gtrsim 0.5)$, the molecule undergoes a continuous phase transition at inverse temperature $\beta_{i}$ given by (5), followed by a discontinuous freezing at $\beta_{d}$. Anticorrelated sequences $(\pi \lesssim 0.5)$ freeze discontinuously at $\beta_{d}$ without intermediate phase. The inverse temperature of abrupt freezing, $\beta_{d}$, turns out to be nearly independent of the sequence, as indicated by the horizontal line at $\beta_{d} \approx 1.23$. The data points show the $\beta_{d}$ 's computed for a few particular sequences with $L=20$.

$\pi$ for two consecutive monomers to be equal. If the instability occurs too close to the entropy crisis of the liquid, it will be prevented by a discontinuous transition at $\beta_{d}$ where a non-trivial solution of the cavity equation (4) appears. This effect can be studied by a numerical solution of this equation using a population dynamics method [14, 17]. Upon increasing the period $L$, we found the transition points to approach a correlation-independent constant $\beta_{d} \approx 1.23 \pm 0.03$ that we believe to be a good approximation in the large $L$ limit, see Fig. 2 .

For ampholytic sequences with a bias to alternation, $\pi \lesssim 0.5$, and for HP-like polymers with a preference for blocks, $\pi \gtrsim 0.5$, there is a continuous glass transition at $\beta_{i}<\beta_{d}$ as predicted by (5). It is followed by a discontinuous freezing transition at lower temperature $\beta_{d}$. In copolymers with opposite correlations, the liquid instability is irrelevant and the freezing transition occurs directly from the liquid globule phase. The grand-canonical phase diagrams for the two scenarios are presented in Fig. 1 for two fixed values of $\pi$.

At the continuous phase transition $\beta_{i}$, the distribution $\rho(\mathbf{p})$ continuously departs from the liquid fixed point $\delta\left(\mathbf{p}-\mathbf{p}^{*}\right)$. The transition is of third order in the free energy, and as usual, the presence of a continuous transition indicates that the replica symmetry is fully broken. The states will organize in an ultrametric hierarchy of clusters with small 'preferences' towards some particular conformations. On lowering the temperature, these preferences grow and result in increasing correlations at 


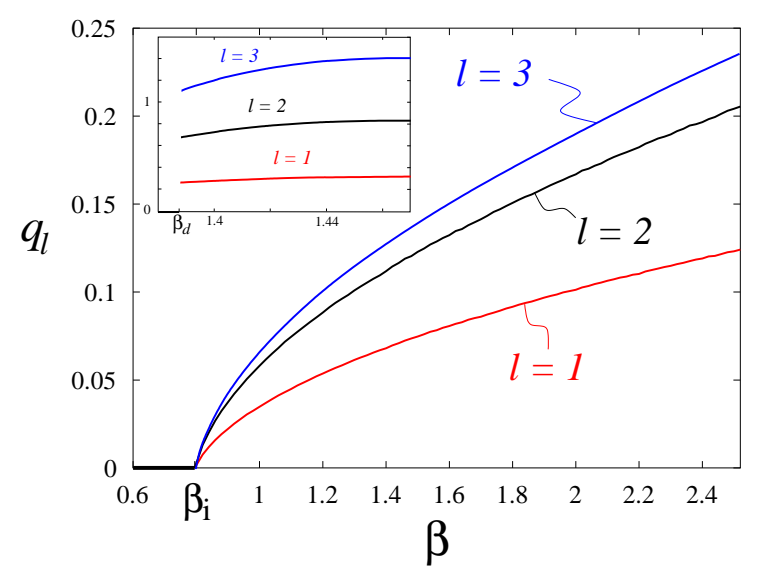

FIG. 3: Temperature dependence of the correlation plateaux $q_{l}$, for three types of correlations with $l=1,2,3$, computed using the cavity method. The main graph shows the continuous transition of strongly correlated HP sequences. The inset displays the discontinuous transition of anticorrelated HP sequences.

larger distances. This effect might be related to the scaledependent freezing predicted in [12, 13] and the intermediate glass phase found in the numerics of [10].

The frozen phase at low temperature is of rather different character. The site probabilities $p_{\alpha}^{(i)}$ are strongly biased towards one particular site-dependent conformation. The pure states correspond to almost frozen conformations and have very small internal entropy, as in the REM.

The freezing phenomenon can be quantitatively described by the correlation plateaux $q_{l}$ defined above. In Fig. 3 we present our analytical results for $q_{l}(T)$ for two sequences with opposite correlations.

What are the modifications of the above mean field picture for realistic (finite-dimensional) models? At least two features must be seriously reconsidered. First, within our approximation, the formation of ordered structures is generally inhibited. Let us consider, for instance, the HP copolymer with highly correlated sequences $\pi \approx 1$. In dimension $D$, at low temperature, the system can separate into A-rich and B-rich regions of linear size $\ell \sim \log (1-\pi)^{1 / D}$, with a frustration energy of order $\ell^{-1}$ per monomer. The phase diagram in Fig. 2 must therefore be modified in the $\pi \approx 1$ (for the HP model) and $\pi \approx 0$ (for the ampholyte) regions due to the emergence of spatially ordered phases. A proper treatment of these phenomena (which are crucial for block copolymers [2]) is beyond the scope of this paper.

Second, the discontinuous dynamical phase transition to the frozen glass phase cannot survive in finite dimensions because of nucleation phenomena. According to the usual scenario of discontinuous glass transitions, we expect it to become a sharp crossover associated with the emergence of very large ('activated') relaxation times [1].

In conclusion, we have described a new approach to heteropolymeric systems. The mean-field approximation is introduced in a well-defined and consistent way, and any further calculation can be checked through simulations on the Bethe lattice. The flexibility of the approach should allow for many applications (e.g., designed sequences) and further refinements (e.g., clusters of sites in the cavity). We considered the case of copolymers with correlated sequences and found that the frozen glass phase can be preceded by a soft glass phase with very different dynamical properties. Since the sequence correlations turned out to be the decisive parameter, it would be interesting to analyze protein sequences in the light of our findings [19].

[1] V. S. Pande, A. Y. Grosberg, and T. Tanaka, Rev. Mod. Phys. 72, 259 (2000).

[2] C. D. Sfatos and E. I. Shakhnovich, Phys. Rep. 288, 77 (1997).

[3] P. G. Wolynes, in Spin Glass Ideas in Biology, D. Stein ed. (World Scientific, Singapore, 1991).

[4] M. Mézard, G. Parisi, and M. A. Virasoro, Spin-Glass Theory and Beyond (World Scientific, Singapore, 1987).

[5] J. D. Bryngelson and P. G. Wolynes, Proc. Natl. Acad. Sci. USA 84, 7524 (1987).

[6] B. Derrida, Phys. Rev. B 24, 2613 (1981).

[7] E. I. Shakhnovich and A. M. Gutin, Biophys. Chem. 34, 187 (1989), Europhys. Lett. 8, 327 (1989). T. Garel and H. Orland, Europhys. Lett. 6, 307 (1988).

[8] T. Garel, L. Leibler, and H. Orland, J. Phys. II (France) 4, 2139 (1994). C. D. Sfatos, A. M. Gutin, and E. I. Shakhnovich, Phys. Rev. E 48, 465 (1993).

[9] V. S. Pande, A. Y. Grosberg, C. Joerg, and T. Tanaka, Phys. Rev. Lett. 76, 3987 (1996).

[10] E. G. Timoshenko, Y. A. Kuznetsov, and K. A. Dawson, Phys. Rev. E 54, 4071 (1996), Phys. Rev. E 55, 5750 (1997), Phys. Rev. E. 57, 6801 (1998).

[11] J.-P. Bouchaud, L. F. Cugliandolo, J. Kurchan and M. Mézard, in Spin Glasses and Random Fields, A. P. Young ed., (World Scientific, Singapore, 1997).

[12] A. Moskalenko, Y. A. Kuznetsov, and K. A. Dawson, Europhys. Lett. 40, 135 (1997).

[13] D. Thirumalai, V. Ashwin, and J. K. Bhattacharjee, Phys. Rev. Lett. 77, 5385 (1996).

[14] M. Mézard and G. Parisi, Eur. Phys. J. B 20, 217 (2001).

[15] J.F. Nagle, Proc. R. Soc. Lond. A 337, 569 (1974), S. Lise, A. Maritan, and A. Pelizzola, Phys. Rev. E 58, R5241 (1998).

[16] P. G. de Gennes, J. Phys. Lett. (Paris) 36, L55 (1975).

[17] A. Montanari, M. Müller, and M. Mézard, in preparation.

[18] P.G. de Gennes, Faraday Discuss. Chem. Soc. 68, 96 (1979).

[19] V. S. Pande, A. Y. Grosberg, and T. Tanaka, Proc. Natl. Acad. Sci. USA 91, 12972 (1994). A. Irbäck and C. Peterson and F. Potthast, Proc. Natl. Acad. Sci. USA 93, 9533 (1996). 